\title{
Report on Aging and Irradiation Theoretical Model Development for Alloy 690 in Grizzly
}

Stephanie A Pitts, B.W. Spencer, T. Feng, J.D. Tucker

March 2018

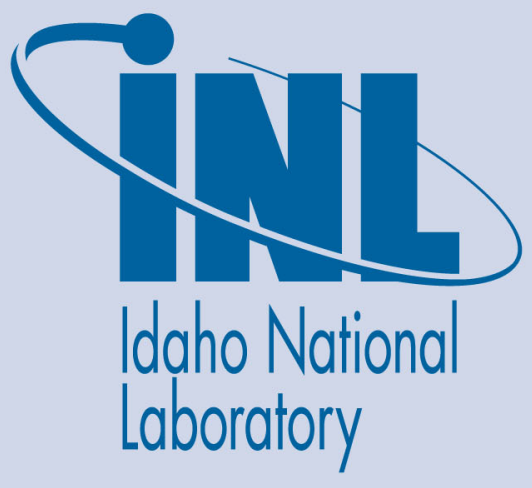

The INL is a U.S. Department of Energy National Laboratory operated by Battelle Energy Alliance 


\title{
Report on Aging and Irradiation Theoretical Model Development for Alloy 690 in Grizzly
}

\author{
Stephanie A Pitts, B.W. Spencer, T. Feng, J.D. Tucker
}

March 2018

Idaho National Laboratory Idaho Falls, Idaho 83415

http://www.inl.gov

Prepared for the

U.S. Department of Energy

Under DOE Idaho Operations Office

Contract DE-AC07-05ID14517 


\title{
Report on Aging and Irradiation Theoretical Model Development for Alloy 690 in Grizzly
}

\author{
S.A. Pitts ${ }^{a}$, B.W. Spencer ${ }^{a}$, F. Teng ${ }^{a, b}$, J.D. Tucker ${ }^{b}$ \\ ${ }^{a}$ Fuels Modeling and Simulation, Idaho National Laboratory \\ ${ }^{b}$ Mechanical, Industrial and Manufacturing Engineering, Oregon State University
}

March 30, 2018

\section{Introduction}

This report describes the current efforts to implement a model to predict the engineering scale behavior of irradiated and aged $\mathrm{Ni}_{2} \mathrm{Cr}$ model alloy and Alloy 690 into the Grizzly software. Grizzly is a multiphysics simulation code under development at Idaho National Laboratory (INL) to simulate aging mechanisms and their effects on the integrity of critical LWR components.

Engineering scale behavior of materials are strongly impacted by the underlying microstructure. The distribution, evolution, and interaction of dislocations and defects within the individual grains dictates how the engineering scale material will perform under different loading and environmental conditions. Mesoscale models can function as a bridge to connect microstructure evolution models to engineering models of the ductile-brittle transition temperature (DBTT) curve and fracture. The toughness of a material, which governs the engineering scale fracture behavior, is a function of both elastic deformation and plastic deformation. While the yield stress measurements capture the elastic contribution to a material's toughness, information about the dislocation movement is required to understand the plastic component of toughness.

Crystal plasticity models predict dislocation density evolution and thereby connect microstructure evolution to engineering scale behavior predictions. These models calculate the plastic strain within a metal as a function of dislocations and other crystal defects, including precipitates and interstitial loops, among others, by tracking dislocation movement and the interaction of these dislocations with the crystal defects.

Within NiCr alloys, long-range ordered (LRO) $\gamma^{\prime}$ precipitates form under aging conditions. These precipitates act as barriers to dislocation motion and either are sheared by dislocations, or act to pin dislocations and prevent dislocation movement. Additional defects, such as stacking fault tetrahedra (SFTs) from irradiation damage, also impede dislocation motion in these alloys. A crystal plasticity mode for $\mathrm{Ni}_{2} \mathrm{Cr}$ model alloy and Alloy 690 is being currently implemented in the Grizzly code at INL.

This report includes a description of the theoretical models incorporated into the Grizzly crystal plasticity code. These theoretical models include mechanisms for dislocation glade and twinning as well as dislocationdefect interaction relationship for both LRO $\gamma^{\prime}$ precipitates and SFTs. We have selected these dislocation models based on the deformation mechanisms observed in experimental micropillar compression tests conducted on the $\mathrm{Ni}_{2} \mathrm{Cr}$ model alloy. The models are then extended to Alloy 690 with ongoing parameter fitting. 


\section{Crystal Plasticity Framework}

Following [1] the crystal plasticity model uses a multiplicative decomposition of the deformation gradient into elastic and plastic components:

$$
\mathbf{F}=\mathbf{F}^{e} \mathbf{F}^{p}
$$

The change in the crystal shape due to dislocation motion is accounted for in the plastic deformation gradient tensor, $\mathbf{F}^{p}$, while the elastic deformation gradient tensor, $\mathbf{F}^{e}$, accounts for recoverable elastic stretch and rotations of the crystal lattice. The evolution of the plastic deformation is given as

$$
\dot{\mathbf{F}}^{p}=\mathbf{L}^{p} \mathbf{F}^{p}
$$

where $\mathbf{L}^{p}$ is the plastic velocity gradient. The plastic deformation gradient rate is used to calculate the increment of the Lagrangian strain. The plastic velocity gradient is defined as the sum of the slip increments from dislocation motion on all of the slip systems. As in the approach of [2] we additively decompose the plastic velocity gradient to account for the various mechanisms of dislocation movement in the $\mathrm{Ni}$ alloy crystal.

$$
\mathbf{L}^{p}=\sum_{N}^{\alpha} \dot{\gamma}_{\text {glide }}^{\alpha} \mathbf{s}_{o}^{\alpha} \otimes \mathbf{m}_{o}^{\alpha}+\sum_{N}^{\beta} \dot{\gamma}_{\text {twin }}^{\beta} \mathbf{s}_{o}^{\beta} \otimes \mathbf{m}_{o}^{\beta}
$$

where $\dot{\gamma}_{\text {glide }}^{\alpha}$ is the slip rate due to dislocation glide and $\dot{\gamma}_{\text {twin }}^{\beta}$ is the dislocation slip rate from twinning. Note that the slip direction and slip plane normal unit vectors, $\mathbf{s}_{o}$ and $\mathbf{m}_{o}$, are defined in the reference configuration. The model for dislocation glide is discussed in Section 2 and the dislocation twinning model is introduced in Section 3.

\section{Dislocation Glide Model}

The plastic dislocation glide slip on each slip system, $\dot{\gamma}_{\text {glide }}^{(\alpha)}$, is connected to the behavior of the mobile dislocations through Orowan's relation, where only dislocation glide is considered.

$$
\dot{\gamma}_{\text {glide }}^{\alpha}=\rho_{\text {mobile }}^{\alpha} b v_{\text {glide }}^{\alpha}
$$

where $\rho_{\text {mobile }}^{\alpha}$ is the mobile dislocation density on each slip system $\alpha, b$ is the burgers vector of the crystal, and $v_{\text {glide }}^{\alpha}$ is the glide velocity of the mobile dislocations. In this implementation we apply a power law expression for the dislocation glide velocity,

$$
v_{\text {glide }}^{\alpha}=v_{o} b\left|\frac{\tau^{\alpha}}{g^{\alpha}}\right|^{1 / m} \operatorname{sign}\left(\tau^{\alpha}\right) \text { if } \tau^{\alpha} \geq g_{o}^{\alpha}
$$

where $v_{o}$ is the initial dislocation velocity, $\tau^{\alpha}$ is the applied resolved shear stress on each slip system $\alpha$, and $g^{\alpha}$ is the slip system resistance or strength which is further defined in Eq (10). As a consequence of the decision to use the slip direction and plane normal unit vectors from the initial crystal orientation, Eq (3), the second Piola-Kirchoff stress is used to determine the applied resolved shear stress.

$$
\tau^{\alpha}=\mathbf{T}: \mathbf{s}_{o}^{\alpha} \otimes \mathbf{m}_{o}^{\alpha}
$$

The constitutive relationship for dislocation glide is based on the Continuum Dislocation Dynamics (CDD) framework, [3], with separate terms used to describe each of the specific physical glide mechanisms in the dislocation evolution rate terms. The rate of the mobile dislocation evolution rate is also fully coupled to the immobile dislocation evolution rate. The mobile dislocation evolution is governed by six terms: each term in the equation represents a specific physical dislocation-interaction mechanism.

$$
\begin{aligned}
\dot{\rho}_{\text {mobile }}^{(\alpha)}= & \dot{\rho}_{\text {generation }}^{(\alpha)}-\dot{\rho}_{\text {mobile-annihilation }}^{(\alpha)}-\dot{\rho}_{\text {locking }}^{(\alpha)} \\
& +\dot{\rho}_{\text {freed }}^{(\alpha)}-\dot{\rho}_{\text {immobile-annihilation }}^{(\alpha)}
\end{aligned}
$$


Mobile dislocation generation is considered to occur by Orowan looping. Dislocation annihilation of two dislocations of opposite sign can occur between two mobile dislocations (second term in Eq. (7)) or between an immobile dislocation and a mobile dislocation (sixth term in Eq. (7)). The annihilation interaction among dislocations is assumed to occur only within a capture radius, $R_{c}$, which is defined as a factor of the burgers vector.

The mobile dislocation evolution is coupled to the immobile dislocation evolution rate through evolution terms for dislocation locks and dislocations freed from locks (third and fourth terms in Eq (7), respectively). These terms also appear in the evolution rate equation for immobile dislocations

$$
\dot{\rho}_{\text {immobile }}^{(\alpha)}=\dot{\rho}_{\text {locking }}^{(\alpha)}-\dot{\rho}_{\text {freed }}^{(\alpha)}-\dot{\rho}_{\text {immobile-annihilation }}^{(\alpha)}
$$

The locked dislocations term, which is negative in the mobile dislocation evolution equation, Eq (7), acts as the source term for the immobile dislocations.

\section{Dislocation Twinning Model}

In low stacking fault metals, twinning is another common mechanism for dislocation motion. Both twinning and microtwinning have been observed in NiCr alloys; microtwinning is described as thin twins only a few atomic planes wide [4]. The presence of secondary and primary $\gamma^{\prime}$ precipitates are considered necessary to initial microtwinning [5]. Karhikeyan et al. have proposed an initial dislocation microtwinning velocity term for use in crystal plasticity [6].

Twinning behavior was observed in the $\mathrm{Ni}_{2} \mathrm{Cr}$ model alloy micropillar compression tests only in the aged specimens; the unaged specimens deformed only by dislocation slip. However, because the observed twins were larger than the microtwin width and because the LRO tertiary $\gamma^{\prime}$ precipitates in the model alloy are much smaller than the secondary $\gamma^{\prime}$ precipitates, the twinning mechanism appears to be better suited to a standard twinning model rather than a microtwinning model. Following Kalidindi's approach, a twinning model is currently being implemented in the Grizzly crystal plasticity model [7].

$$
\dot{f}_{\text {twin }}^{\beta}=\frac{\dot{\gamma}}{\gamma_{\text {twin }}}\left(\frac{\tau^{\text {beta }}}{g_{\text {twin }}^{\text {beta }}}\right)^{1 / m} \text { if } \tau^{\beta}>0
$$

This model tracks the evolution of the deformation twin volume fraction, $\dot{f}^{\beta}$ instead of the twin slip rate.

\section{$4 \quad$ Hardening Models}

Physically-based frameworks write the constitutive slip system resistance equation as a function of dislocation and defect densities within the crystal [8]; both defects and other dislocations act as barriers to dislocation motion [9]. In this model the resistance of the slip systems to dislocation motion is considered as the additive sum of the physical barriers to dislocation motion, including dislocation forests, aged long-range ordered precipitates, and irradiation defects.

$$
g^{(\alpha)}=g_{o}^{(\alpha)}+g_{\text {dislocations }}^{(\alpha)}+g_{\text {precipitates }}^{(\alpha)}+g_{\text {defects }}^{(\alpha)}
$$

where $g_{o}^{(\alpha)}$ represents the intrinsic lattice friction, $g_{\text {dislocations }}^{(\alpha)}$ models the hardening of slip systems due to the accumulation of other dislocations on all of the slip systems, $g_{\text {precipitates }}^{(\alpha)}$ accounts for the hardening due to the long-range ordered precipitates, and $g_{\text {defects }}^{(\alpha)}$ is used to capture the effect of irradiation lattice defects on the slip system hardening. The first two terms in Eq (10) are discussed below and the latter two terms are discussed in Section 5 and Section 6, respectively. 


\subsection{Intrinsic Lattice Friction}

The intrinsic resistance of a crystal to dislocation motion is due to friction forces, and the measure of this resistance is termed the Peierls strength of the material. Because $\mathrm{NiCr}$ alloys are low stacking fault materials compared to pure $\mathrm{Ni}$, the spreading of the dislocation core will play a role in the intrinsic lattice friction of the alloy. Following the work of [10], we assume as a reasonable estimate for the Peierls strength

$$
g_{o}^{(\alpha)}=10^{-4} \cdot \mu
$$

where $\mu$ is the shear modulus of the alloy. This assumption allows for easy extension of the crystal plasticity model parameter to different alloy systems without the precise lattice spacing dimensions required by the classical Peierls-Nabarro model.

\subsection{Dislocation Forest Hardening}

The slip system resistance due to dislocation forest-type accumulation on all slip systems is modeled with a modified Bailey-Hirsch approach following [11]:

$$
g_{\text {dislocations }}^{(\alpha)}=\alpha_{m b h} b \mu \sqrt{\sum_{\beta} \Omega^{(\alpha \beta)}\left(\rho_{\text {mobile }}^{(\beta)}+\rho_{\text {immobile }}^{(\beta)}\right)}
$$

where the hardening is termed self-hardening when $\alpha=\beta$ and latent-hardening when $\alpha \neq \beta$. The coefficient $\alpha_{m b h}$ is a fitting parameter, $b$ is the burgers vector, $\mu$ is the shear modulus of the material, and $\Omega$ is the interaction matrix containing the matrix of self- and latent-hardening parameters. For simplicity, in this work we assume all components of the interaction matrix to be unity.

\section{$5 \quad$ Hardening from Aging Precipitates}

The hardening due to LRO $\gamma^{\prime}$ precipitates is accounted for with two separate mechanisms: Anti-phase boundary (APB) particle shearing and Orowan looping.

$$
g_{\text {precipitates }}^{(\alpha)}=\alpha_{\text {apb }} g_{\text {apb }}^{(\alpha)}+\alpha_{\text {looping }} g_{\text {looping }}^{(\alpha)}
$$

In establishing the relationship among the two different shearing mechanisms, we assumed that a given dislocation could either shear a precipitate via APB shearing or loop around a precipitate; thus we enforced the condition

$$
\alpha_{\text {apb }}+\alpha_{\text {looping }}=1
$$

Through fitting to experimental hardness testing data for $\mathrm{Ni}_{2} \mathrm{Cr}$ aged for 10,000 hours [12], we determined that the majority of hardening occurs due to APB shearing, Eq (15). Combining Eqs (13) and (14), we determined $\alpha_{a p b}$ is 0.95 and $\alpha_{\text {looping }}$ is 0.05 for the $\mathrm{Ni}_{2} \mathrm{Cr}$ model alloy.

The following hardening mechanisms were selected from the review provided by Kozar et al for the small tertiary $\gamma^{\prime}$ precipitates experimentally observed in the $\mathrm{Ni}_{2} \mathrm{Cr}$ alloy. The presence of primary and secondary $\gamma^{\prime}$ precipitates in Alloy 690 may require the addition of other hardening mechanisms beyond the two presented in $\mathrm{Eq}(13)$.

\subsection{Tertiary Anti-Phase Boundary Precipitate Shearing}

The $\mathrm{Ni}_{2} \mathrm{Cr}$ model alloy only demonstrates tertiary LRO precipitates, under $10 \mathrm{~nm}$ in size. Based on this size, we have implemented a model for weakly coupled APB shearing [13]. The effective spacing between two LRO precipitates is modeled in a manner that accounts for the radii of the precipitates.

$$
g_{a p b}^{(\alpha)}=\frac{\gamma_{a p b}}{2 b}\left[\left(\frac{\gamma_{a p b} d_{s}}{\mu}\right)^{1 / 2} \frac{d_{s}}{b L_{s}}-\frac{\pi}{4}\left(\frac{3 \pi f}{8}\right)\right]
$$


where $\gamma_{a p b}$ is the energy of the anti-phase boundary, $b$ is the burgers vector, $d_{s}$ is the mean planar diameter of the precipitate, $\mu$ is the shear modulus, $L_{s}$ is the effective spacing between precipitates, and $f$ is the volume fraction of the tertiary precipitates. The effective spacing between precipitates takes into account the diameter of the precipitates, [13]:

$$
L_{s}=\left(\frac{8}{3 \pi f}\right)^{1 / 2} d_{s}-d_{s}
$$

The weakly coupled terminology refers to the ability of the precipitate to contain only a single dislocation partial at a time; larger precipitates which can contain both partials of a dislocation are said to undergo strongly coupled APB shearing.

\subsection{Orowan Looping of Precipitates}

For those dislocations which loop around a precipitate instead of shearing through the precipitate, we have applied an Orowan looping strength term.

$$
g_{\text {looping }}^{(\alpha)}=\frac{\mu b}{L_{s}}
$$

As in the APB shearing term, Eq (15), the effective spacing between two precipitates is implemented [13] using Eq (16).

\section{Irradiation Defects Hardening}

The dominant irradiation damage defects in copper, another FCC material, are SFTs; therefore we assume that SFTs are a common irradiation damage defect in the NiCr alloys. Following Arsenlis et al, we model the contribution of the SFTs with a modified dispersed barrier hardening model [14].

$$
g_{\text {precipitate-defects }}^{(\alpha)}=\alpha_{d b} b \mu \sqrt{N_{s f t} d_{s f t}}
$$

where $\alpha_{d b}$ is a fitting coefficient on the order of $0.2[15], b$ is the burgers vector, $\mu$ is the shear modulus, $N_{s f t}$ is the number density of the SFTs and $d_{s f t}$ is the average size of the SFTs. The evolution of the SFT number density and size, as a result of the cutting the SFTs into two smaller defects by dislocations shearing the defect, (will be) modeled using the rate equations introduced by [14].

\section{Micropillar Simulation}

To compare the crystal plasticity simulations to the experimental micropillar compression tests on the $\mathrm{Ni}_{2} \mathrm{Cr}$ model alloy, a micropillar geometry is being used to both compare the simulation results to the experimental data and determine appropriate boundary conditions for a smaller crystal plasticity simulation geometry. Initial micropillar compression simulations, performed on a coarse mesh, were loading in the [100] direction of the crystal lattice. Elastic constants for Alloy 690 were used in this simulation.

The effective stress response and the mobile dislocation density from the initial micropillar compression simulation are shown in Figure 1. In the [100] loading direction, an FCC crystal has 8 activated slip systems. All 8 slip systems demonstrated similar evolution trends; the active slip system [110](11̄î) is used as an example for all 8 active slip systems. 


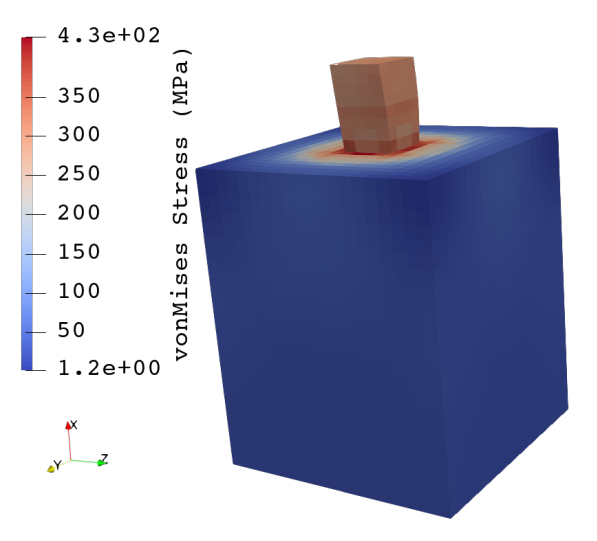

(a) Effective Stress in Micropillar

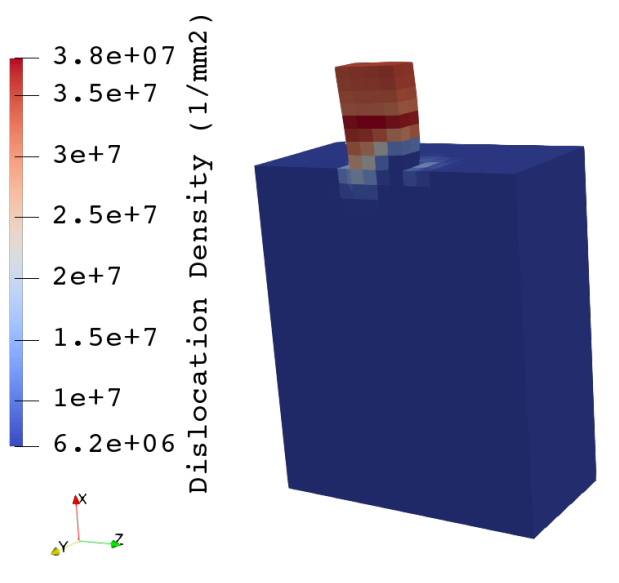

(b) Center View [110](11̄̄) Mobile Dislocation Density

Figure 1: The initial micropillar model simulation shows displacement in the loading direction concentrated in the micropillar with a small component of the displacement borne by the substrate. Evolution of the mobile

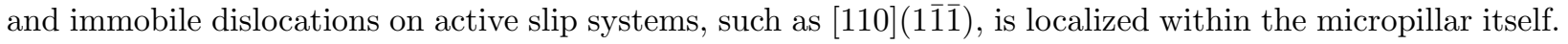

These initial simulation results confirm our expectation that plastic deformation from dislocation evolution is confined to the micropillar while the substrate contributes to the overall elastic response. A full comparison set of crystal plasticity simulation results against experimental data, including loading orientations along the [001] and [111] slip directions, will be discussed in a future report.

\section{Summary and Future Work}

As with any modeling effort, these developments to the crystal plasticity simulation capabilities are accompanied by experimental efforts to inform and validate the software modeling development work. This report details the theoretical and computational model development efforts to implement a crystal plasticity model in Grizzly for aged model Ni-Cr alloy and irradiated Ni-Alloy 690. Verification and validation efforts to compare the Grizzly crystal plasticity predictions to experimental data are on-going and will be described as a component of the final report for this project.

\section{References}

[1] Robert J Asaro. Crystal plasticity. Journal of Applied Mechanics, 50(4b):921-934, 1983.

[2] Xianping Wu, Surya R Kalidindi, Carl Necker, and Ayman A Salem. Prediction of crystallographic texture evolution and anisotropic stress-strain curves during large plastic strains in high purity $\alpha$ titanium using a taylor-type crystal plasticity model. Acta Materialia, 55(2):423-432, 2007.

[3] Dongsheng Li, Hussein Zbib, Xin Sun, and Mohammad Khaleel. Predicting plastic flow and irradiation hardening of iron single crystal with mechanism-based continuum dislocation dynamics. International Journal of Plasticity, 52:3 - 17, 2014.

[4] L Kovarik, RR Unocic, Ju Li, P Sarosi, C Shen, Y Wang, and MJ Mills. Microtwinning and other shearing mechanisms at intermediate temperatures in ni-based superalloys. Progress in Materials Science, 54(6):839-873, 2009.

[5] RR Unocic, L Kovarik, C Shen, PM Sarosi, Y Wang, J Li, S Ghosh, and MJ Mills. Deformation mechanisms in ni-base disk superalloys at higher temperatures. Superalloys, 8:377, 2008. 
[6] S Karthikeyan, RR Unocic, PM Sarosi, GB Viswanathan, DD Whitis, and MJ Mills. Modeling microtwinning during creep in ni-based superalloys. Scripta Materialia, 54(6):1157-1162, 2006.

[7] Surya R Kalidindi. Modeling anisotropic strain hardening and deformation textures in low stacking fault energy fcc metals. International Journal of Plasticity, 17(6):837-860, 2001.

[8] F. Roters, P. Eisenlohr, L. Hantcherli, D. D. Tjahjanto, T. R. Bieler, and D. Raabe. Overview of constitutive laws, kinematics, homogenization and multiscale methods in crystal plasticity finite-element modeling: Theory, experiments, applications. Acta Materialia, 58(4):1152-1211, 2010.

[9] John P Hirth and Jens Lothe. Theory of dislocations. 1982.

[10] Peter Szelestey, Marco Patriarca, and Kimmo Kaski. Computational study of core structure and peierls stress of dissociated dislocations in nickel. Modelling and Simulation in Materials Science and Engineering, 11(6):883, 2003.

[11] Tetsuya Ohashi. Numerical modelling of plastic multislip in metal crystals of fcc type. Philosophical Magazine A, 70(5):793-803, 1994.

[12] George A Young and Daniel R Eno. Long range ordering in model ni-cr-x alloys. In Proceedings of the International Symposium Fontevraud 8 on the Contribution of Materials Investigations and Operating Experience to LWRs Safety, Performance and Reliability, 2015.

[13] RW Kozar, A Suzuki, WW Milligan, JJ Schirra, MF Savage, and TM Pollock. Strengthening mechanisms in polycrystalline multimodal nickel-base superalloys. Metallurgical and Materials Transactions A, 40(7):1588-1603, 2009.

[14] A. Arsenlis, B. D. Wirth, and M. Rhee. Dislocation density-based constitutive model for the mechanical behaviour of irradiated Cu. Philosophical Magazine, 84(34):3617-3635, 2004.

[15] GR Odette and D Frey. Development of mechanical property correlation methodology for fusion environments. Journal of Nuclear Materials, 85:817-822, 1979. 